\title{
Introduction to Self-attachment and its Neural Basis
}

\author{
Abbas Edalat \\ Algorithmic Human Development Group \\ Department of Computing, Imperial College London \\ Email: a.edalat@imperial.ac.uk
}

\begin{abstract}
We introduce the notion of self-attachment which, based on an interdisciplinary set of concepts, proposes a new psychotherapeutic technique. The underlying ideas include findings and paradigms in developmental psychology and neuroscience, neuroplasticity and long term term potentiation, fMRI studies on human bond making, ethology and psychology of religion and experiments in energy based artificial neural networks. The proposed self-attachment therapeutic technique is distinguished by its intervention to create an internal and passionate affectional bond within the individual between the "adult self", representing the logical and cognitive faculty, and the "inner child", representing the unregulated and undeveloped emotional circuits. The aim is to create more optimal circuits for emotional regulation. The proposed self-attachment protocols internally emulate within the individual the interactions of a good enough primary care-giver and child in order to moderate the child's arousal level, minimise its negative affects and maximize its positive affects. These interactions are assumed, in developmental neuroscience and in developmental psychology, to be the basis of secure attachment of children with their parents, which leads to an optimal regulation of neurotransmitters, hormones, and the emotional dynamics of the individual. We report on several case studies of this technique in recent years. Finally, we propose a simple mathematical model to capture the impact of self-attachment protocols using the notion of strong patterns in energy based neural networks and employ a recently developed mathematical model to examine the impact of self-attachment using emotional and cognitive neural pathways for decision making. ${ }^{12}$
\end{abstract}

\section{INTRODUCTION}

Psychotherapy, or the talking cure, as introduced by Freud was at first essentially a cognitive form of therapy. The objective in the course of psychoanalytical treatment of a client was to bring into consciousness, by gaining insights through interpretation of free association and dreams as well as analysis of transference and other defences, what had been repressed in early childhood, which was assumed, based on Freud's drive theory of psychic development, to be the root cause of neurosis or psychological disorder. In the second half of the past century, there was a shift away from Freud's drive theory toward an understanding of the development of human psyche in relation to significant figures in the early environment during childhood. This new focus was prompted by the work of object-relation psychoanalysts, but it is generally agreed that John Bowlby's attachment theory, recognised in developmental psychology as a scientific paradigm in the latter part of the 20th century, had the highest impact in this direction including on

\footnotetext{
${ }^{1}$ This is an expanded version of the paper with the same title in the Proceedings of IEEE's International Joint Conference on Neural Networks, IJCNN, Killarney, Ireland, July 12 - 17, 2015.

${ }^{2}$ Other papers realted to self-attachment can be obtained from the web-page of the Algorithmic Human Development Group: http://humandevelopment.doc.ic.ac.uk/
}

psychoanalysis in general; see [1] for a comprehensive study of the relation between psychoanalysis and attachment theory.

In more recent decades, we can in fact see, in the words of Allan Schore, a paradigm shift toward an emphasis on affect regulation in psychotherapy [2]. This trend and paradigm shift can be inferred in the development of schema therapy [3] whose original roots are in cognitive behavioural therapy, the most widely used psychotherapeutic method in the West. A main intervention in schema therapy, in line with the paradigm shift described above, is to address and tackle dysfunctional emotional patterns or schemas, formed in an early stage of development in an individual.

In this paper, we propose the notion of self-attachment, which, in a broad sense of the term, can be regarded as a type of self-directed behaviour and interaction that employs a real or imaginative object of devotion and affection and is practiced regularly in order to regulate emotions and harmonise social interaction. We submit that some form of self-attachment, in this sense of the word, can be identified in children's emotional development as well as in religious practice. There is also evidence, we will argue, for self-attachment in ethology.

The so-called self-attachment technique, proposed as a new psychotherapeutic method, has been developed since 2010 in the UK and, based on four case studies, was first reported at the Institute of Psychiatry in London in May 2013 [4]. Since then the proposed protocols for self-attachment have been formulated and have become the basis of a number of new case studies conducted by several independent professional psychotherapists. It is hypothesised that this technique creates optimal circuits that counter the sub-optimal pathways created in early development.

The purpose of this paper is to outline the inter-disciplinary theoretical foundation of this technique focusing on its hypothesised neural basis, briefly provide some evidence for selfattachment from ethology, human biology and anthropology, present the general line of intervention it makes and its hypothesised neural correlates, give a short description of the protocols involved and describe a simple mathematical model that can capture the core concept of this proposed method.

The paper is organised as follows. In Section II, we explain the central tenets of attachment theory and its neural basis. In Section III, we describe the common patterns in three fMRI studies on "falling in love". In Section IV, evidence for self-attachment, in the broad sense of the term, is presented from psychology and ethology. In Section V, we describe strong patterns of energy based neural networks as a model for behavioural and attachment prototypes. In Section VI, we briefly describe the proposed self-attachment therapeutic 
technique and finally in Section VII, we construct a neural model for self-attachment.

\section{ATTACHMENT THEORY}

As the name self-attachment implies, this technique has been grounded on Attachment Theory, a fundamental scientific paradigm in developmental psychology as pioneered by John Bowlby and developed further by Mary Ainsworth and Mary Main in the second half of the previous century.

Bowlby hypothesised that the kind of emotional attachment children develop with their primary caregivers will become the foundation of what he called the "internal working model", a cognitive framework with which the child and later the adult interprets the social world and predicts the outcome of her/his behaviour in view of past memory. Thus, through the internal working model, the early attachment types of a child have a great impact on its future emotional and personality development as an adult in social and intimate relationships.

In its current state, attachment theory classifies the type of emotional attachment of one year old toddlers to their primary care givers into one of four types of attachments. A very simplified description of these types are presented here: (1) Securely attached, which results from the child interacting with a "good enough" primary care giver, who is sensitive to the emotional needs of the child and responds quickly and appropriately to its distress signals. The other three types are regarded as insecure attachments as follows: (2) Avoidantly attached, which results from the behaviour of a primary care giver who mostly rebuffs the child's signals of emotional distress. (3) Ambivalently or anxiously insecure, reflecting a primary care giver who acts inconsistently, and finally, (4) Disorganisedly insecure which is brought about when the primary caregivers have significant psychological disorders going back to their own childhoods and act either in a frightening way or alternatively are frightened by the distress signal of the children.

The Strange Situation Procedure, a 20 minutes experiment involving a mother, her one year old toddler, a stranger and an unfamiliar room, was developed by Mary Aisworth in 1970's to elicit the basic classification of attachment types. It has been repeated across different cultures mostly in the western world and the results indicate mostly similar distribution of attachment types among the toddlers in different societies [5].

\section{A. Neurobiology of secure attachment}

There is now a significant volume of information on how an infant's secure attachment, in interaction with the good enough mother or primary care giver, is reflected in the specific growth of the infant's brain. A detailed exposition of the subject, which collects together the work of many researchers, is presented in [6] and in[7]. Visual experience is thought to play a paramount role in the emotional development of the infant and its emotional attachment to the mother in the first year, the so-called practising year. Through intense mutual gaze, the infant-mother dyad is established, creating a framework for transmission of mutual influence and inter-communication. The mother is attuned to and resonates with the internal state of the infant at rest and is able to contingently fine tune her affective stimulation with the infant's dynamically activated, deactivated or hyperactivated state. By her facial expressions, singing, dancing and play, she maximises the positive affects and minimises the negative affects of the infant. Through this right brain-right brain interactions, focused on facial expressions, synchronised interactions between the infant and the mother are coordinated, giving rise to a mutual regulatory system of arousal in which the mother and the infant can move back and forth from low to high but modulated levels of arousal. It is assumed that this self-regulatory process in the infant-mother dyad is the mechanism that mediates the infant's attachment and bond formation with the mother.

The infant's right hemisphere is intensely involved in the visual interactions with the mother and is used as a template for imprinting what it receives from the mother's emotionregulatory right cortex. This leads to entrenched circuits in the infant's right brain that reflect the mother's right brain activities and will be used in turn to regulate the infant's emotions. The mutual and intense gaze, facial expressions, mirroring and resonance between the infant and the mother release endogenous opiates inducing pleasure and release of catechlamine dopamine from the ventral tegmental resulting in dopaminergic-driven arousal and dopamine-mediated elation. High levels of dopamine secretion results in a rapid growth rate of the infant's brain by accelerating the transcription of genes and DNA synthesis and regulating dendritic growth.

Schore quotes the following assertion from developmental psychoanalysis: "It is the emotional availability of the caregiver in intimacy which seems to be the most central growthpromoting feature of the early rearing experience" [8]. He then summarises the significance of these early infant-mother interactions: "This mutual regulatory system is adaptive in that it allows for the arousal level of the child's developing nervous system to be rapidly recalibrated against the reference standard of the mother's." [6, p. 12]

A large volume of research in neuroscience highlights that the orbital prefrontal cortex (OFC), which is densely connected to the limbic system as well as to the cortex and is developed in the last months of the first year, plays the crucial role in complex self-regulation and attachment processes. The optimal growth of the OFC in the securely attached toddler allows delayed response based on stored representation rather than immediate information in the environment. The infant acquires a mental representation of the mother that can increasingly be used in her absence as well. Schore concludes: "As a result, this prefrontal system can now generate interactive representations-non-verbal internal working models of the infant's transactions with the primary attachment figure that dyadically maximize positive and minimise negative affect."

In the second year, the so-called socialising period, the toddler, who can now walk around, is now regularly prohibited from engaging in dangerous situations or anti-social behaviour. This misattunement with the mother results in the toddler's painful experience of shame and the break-up of homoeostasis with the mother. The warm and appropriated intervention of the mother, however, leads to reattunement. As a result of repeated misattunement and reattunement with the mother, the securely attached toddler gradually learns that the homoeostasis disruption with the mother can always be reestablished and is thus able to regulate its painful shame. Schore evaluates the maturation of the infant's brain as a 
result of these disruption-repair transactions between the infant and the mother as follows: "These experiences trigger specific psychobiological patterns of hormones and neurotransmitters, and the resultant biochemical alterations of the brain chemistry influence the experience-dependent final maturation of the orbitofrontal cortex." In this way, the child's brain becomes focused on exploring the world: in the words of Julian Ford, a "learning brain" develops [9], a concept that fits with the idea of "knowledge instinct" [10]. The OFC is also involved in internal regulation of states by its connections to the hypothalamus, which allows cortical containment of the autonomic sympathetic and parasympathetic somatic reactions induced by emotions [6].

\section{B. Neurobiology of insecure attachment}

While the mother of a securely attached child responds quickly and appropriately to the child's distress signals and expresses a great deal of warm affection on reunions, the primary caregiver of an insecurely attached child is inaccessible to the child on reunions, and fails to provide adequate, consistent and unconditional affection to the child. This primary caregiver may have an aversion to physical contact and may hesitate or even rebuff the child's distress signals, which the child can only interpret as an assault. As a result the child shows no interest in proximity seeking with this primary caregiver and the normal process of attachment bonding is severely disrupted, which in turn leads to failure of the regulatory mechanism and disturbance in the limbic activity and hypothalamic dysfunction. The primary caregiver's failure to engage quickly and appropriately in repairing the homoeostasis disruption traumatises the infant and results in defects in the development of the OFC and in biochemical dysregulation and toxic brain chemistry of the infant. In this way, the child's main focus will be how to avoid further trauma: in the words of Julian Ford, a "survival brain" develops [9].

It is believed that whereas in an avoidantly attached child the sympathetic nervous system becomes disregulated, in an anxiously attached child the parasympathetic nervous system is impaired and in a disorganisedly attached child both systems become disfunctional. See [7] for an overview of the subject.

Here, we highlight the results in [11], where the authors examined amygdala activation, feelings of irritation, and the use of excessive force as indicated by grip strength during exposure to infant crying and scrambled control sounds in 21 women without children. Individuals with insecure attachment representations, based on Berkeley Adult Attachment Interview, showed heightened amygdala activation when exposed to infant crying compared to individuals with secure attachment representations. In addition, insecure individuals experienced more irritation during infant crying and used more excessive force than individuals with a secure representation.

\section{NEUROIMAGING OF BOND MAKING}

In [12], Bartles and Zeki reported on fMRI studies of passionate/romantic love. Six men and and eleven women, who were passionately in love with their partners, stared at photos of their partners or at photos of their friends for about 17 seconds. The conclusion was that looking staringly at a the photo of a partner increased activation of the dopaminergicrelated brain areas such as the caudate nucleus and putamen.
These findings were reinforced by fMRI experiments reported in [13] by Aron et al. on partners in the early stage of passionate love, which showed increased activity in dopaminerich subcortical brain areas, the ventral tegmental area and caudate nucleus.

In [14], Bartles and Zeki conducted an fMRI experiment, similar to that in [12], on twenty mothers when each stared at the photos of their own child, another child of the same age with whom they were acquainted with, their best friend, and photographs of another person they were acquainted with. There was increased activity in the dopaminergic-rich subcortical brain areas (caudate nucleus, putamen, subthalamic nucleus, periaqueductal gray, substantia nigra, and lateral thalamus). There were certainly some specific differences in activation patterns in romantic love and maternal love, in particular the activation of the periaqueductal (central) gray matter (PAG) was observed in maternal but not passionate. However, the conclusion was that there are neural correlates common to both maternal and romantic love, which are based on increased activation of the the dopaminergic rich subcortical regions of caudate nucleus and putamen.

While the above fMRI studies were related to bond making in human beings, Uffe Schjoedt et al. in [15] investigated how performing religious prayers changed the neural activity in a group of Danish Christians. They had five tasks to perform that included two prayers, the Lord's Prayer, as a highly formalized prayer, and a personal prayer as an improvised prayer. The participants all reported that they were strong believers in God's existence and regularly prayed. The result was the activation of the caudate nucleus in the participants which supports the hypothesis that religious prayer is capable of stimulating the dopaminergic system of the dorsal striatum in practicing individuals. This conclusion is consistent with research on the human striatum indicating that repeated behaviours which are expected to elicit future rewards evoke activity in the dorsal striatum. Furthermore, the authors say that in a related study, "we found no significant caudate activation of self-reported religious persons, who did not pray regularly". While one can argue that prayer involves interaction with an abstract idea of some deity in one form or the other, as far as the brain activity is concerned, it is no different than "normal interpersonal interaction". On this subject, Uffe Schjoedt, the lead author of the article, writes [16]: "Importantly and somewhat contrary to the widespread assumption that communicating with God constitutes a unique experience reserved for believers, our findings suggest that praying to God is comparable to normal interpersonal interaction, at least in terms of brain function. Praying, it seems, is subserved by the basic processing of our biologically evolved dispositions like other complex cultural phenomena, in this case the evolved human capacity for social cognition."

\section{EVIDENCE FROM PSYCHOLOGY AND ETHOLOGY}

In a well known sentence in his last paper before he passed away, Bowlby summarised the paradigm of bond making: "Falling in love is the formation of a bond whereas loving is the process of maintaining the bond. Although Bowlby was addressing the process of bond making between humans, we can infer from the fMRI experiments on religious believers that his description of attachment bond can be extended beyond the 
case of two real human beings. In fact, there is evidence for certain forms of self-attachment, in a general sense of the word, by means of an inanimate or abstract object in developmental psychology and in the psychology of religion.

The concept of transitional object was introduced in 1953 by Donald Winnicott, the British paediatrician and childpsychoanalyst, to describe "comfort objects" such as pillows, blankets and soft toys that a child becomes intensely and passionately attached to [17]. According to him, for toddlers with good enough mothers, these attachments to transitional objects play a key role in ego development: the child projects the comforting properties of a good enough mother to the inanimate object which, unlike the mother who can temporarily disappear, is always under the control of the child whether the mother is present or not. By practicing and interacting with the transitional object, a mother substitute, the child then acquires the capacity for self-soothing by internalising the good enough mother and is able to withstand increasingly longer absences of the mother. Later researchers have formulated a number of key functions of transitional objects, including separationindividuation, libidinal object constancy, capacity for object relation and empathy and symbolisation and creativity [18]. In 1958, Winnicott also introduced another concept, namely the "capacity to be alone" [19]. His article starts by asserting that "I wish to make an examination of the capacity of the individual to be alone, acting on the assumption that this capacity is one of the most important signs of maturity in emotional development." He theorised that this capacity can be developed in children who have good enough mothers and have thus, in psychoanalytical terminology, introjected or internalised a good object in their inner psychic world. In Winnicott's view this capacity can however only take shape by the experience of "being alone, as an infant and a small child, in the presence of the mother". According to him, only by acquiring the capacity to be alone the child can discover its "true self" in contrast to "a false life based on reactions to external stimuli". It is based on this self-discovery that the child's capacity to be alone has been proposed as the foundation of independence and creativity later in life [20], and can thus be regarded as an advanced form of secure attachment.

William Meissner, a Jesuit and a psychoanalyst, has used the notion of transitional object to explain the psychology of praying [21]. Meissner writes the following about the individual believer's praying: "The God he prays to is not ultimately the God of the theologians or of the philosophers, nor is this God likely to be in any sense directly reconcilable with the God of Scripture. Rather, the individual believer prays to a God who is represented by the highly personalised transitional object representation in his inner, private personally idiosyncratic belief system." This view is in line with that by Uffe Schjoedt quoted in the previous section.

We can regard interactions with transitional objects, whether used by young children or employed, in the sense of Meissner, in religious experience, as forms of self-attachment. In a broad sense of the word, self-attachment can be regarded as a form of passionate self-directed behaviour and interaction which employs a real or imaginative object of devotion and affection, and is practiced by an individual regularly to help regulate emotions. In these experiences whether by a child or an adult, a passionate and intense attachment with an inanimate or an abstract object is established and is followed up by various forms of rituals which would overall allow for some regulation of the individual's strong emotions and harmonise his or her social interactions.

We can identify Bowlby's two components of "falling in love" as the beginning of the process of bond making, and "loving", as the process of maintaining the bond, both in children interacting with their transitional objects and in adults in religious experience and religious conversion. In both cases an internal affectional bond is first established with the beloved object and then the bond is maintained by various forms of rituals. One can therefore regard certain aspects of religious practice of individuals as having many parallels with the general idea of self-attachment, i.e., self-directed behaviour mediated by an internal bond with an attachment object such as a deity which aims to regulate strong emotions and harmonise social relationships of the individual. Such a viewpoint is supported by the writings of Karen Armstrong, a prominent researcher in the field of religion [22]. It is also in line with the classic findings of William James in his well respected study of religious experience and religious conversion [23].

There is in addition evidence in ethology that rudimentary forms of soothing self-directed behaviour have been biologically adaptive in higher primates. We recall that Bowlby in the late 50's and in the 60's worked very closely with the leading ethologist Harry Harlow and in particular used Harlow's experiments on infant Rhesus monkeys with surrogate dummy mothers, consisting of either milk bottle mothers or cloth and wire mothers, to find further support for attachment theory [24].

In more recent times, some new patterns of behaviour have been observed and documented about isolated infant monkeys. Experiments, unethical by today's standards, on Rhesus monkeys, isolated at birth, show that after six months in isolation they exhibit a type of self-directed behaviour, such as self-orality, clasping a limb or rocking, which are considered soothing and comforting since they are precisely the kind of actions that would have been carried out on the monkey infant by the monkey's mother if she had been present [25]. These self-directed soothing behaviours must have biological underpinning as they are not learnt and give more evidence that self-attachment is a particular component of the attachment system discovered by Bowlby.

\section{Modelling Attachment Types in Neural Nets}

Attachment Theory has been studied in computer science and Artificial Intelligence by Dean Peters and his collaborators; in particular in [26] a reactive agent architecture has been designed to model infant's attachment types. In [27], the discrete energy based neural network usually referred to as the Hopfield model, but was previously conceived in its continuous form in [28], has been used to design an attachment model for robots. In [29], an arousal based model of attachment types has been constructed using Deep Belief Networks.

In the field of psychology, the three academic psychiatrists Lewis, Amini and Lannon used artificial neural networks to highlight, in a non-technical language, that our attachment types and key emotional attitudes in relation to others are sculpted by limbic attractors as a result of repeated exposure to 
similar patterns of interactions in childhood, which will then profoundly impact our emotional world for the rest of our lives[30, p. 132-144].

A similar argument about repetition of a pattern has been implicitly made by three sociologists, Smith, Stevens and Caldwell in [31, p. 222], who proposed the Hopfield network to model behavioural prototypes (including addictions), and working models in attachment theory. Cognitive and behaviourally patterns (including for example substance abuse, habitual physical exercise, gambling and eating), they argued, control the neurophysiology underlying feelings associated with distress and can be considered as: prototypes, deeply learned patterns of thought and social activity".

On the other hand, any form of psychotherapy can be regarded as an effort to counter some dysfunctional behavioural or cognitive or affective prototypes with some more adaptive prototypes. Thus a model in artificial neural networks of how prototypes are created and become sculpted in the brain can be useful in psychotherapy as well as psychology in general.

In order to represent a deeply or repeatedly learned pattern or prototype, the notion of a strong pattern of the discrete Hopfield network was introduced in [32], [33] as a pattern that has been multiply stored in the network. These patterns result in strong and stable attractors with a large basin of attraction.

Recall that in a Hopfield network of say $N$ nodes, each node $i=1, \ldots, N$ takes one of two values $x_{i}= \pm 1$. Suppose that $p$ patterns $\xi^{\mu}$ are to be stored with degrees $d_{\mu}$ for $\mu=$ $1, \ldots p$. In terms of the generalised Hebbian rule the symmetric synaptic weights $w_{i j}$ between nodes $i$ and $j$ are now computed according to the following rule:

$$
w_{i j}=\frac{1}{N} \sum_{\mu=1}^{p} d_{\mu} \xi_{i}^{\mu} \xi_{j}^{\mu}
$$

for $i \neq j$ with $w_{i i}=0$ for $1 \leq i, j \leq N$. It was shown in [32] that the energy of the strongest pattern stored in a network decreases linearly with the degree (multiplicity) of the strong pattern. For simplicity let's focus on the case that $\xi_{i}^{\mu}$ for $\mu=1, \ldots p$ and $i=1, \ldots, N$ are random with values \pm 1 each with probability $1 / 2$, and that there is a single strong pattern $\xi^{1}$ with $d_{1}>1$ and all other patterns are simple, i.e., $d_{\mu}=1$ for $\mu>1$. Then, the distribution of error in the stability of $\xi^{1}$ when $N \rightarrow \infty$ and $d_{1} \ll p$ is given by (cf. [32]):

$$
\begin{aligned}
& \left.\operatorname{Pr}_{e r}=\sqrt{N /(2 \pi p}\right) \int_{d_{1}}^{\infty} e^{-N x^{2} /(2 p)} d x \\
& =\frac{1}{2}\left(1-\operatorname{erf}\left(d_{1} \sqrt{N / 2 p)}\right)\right.
\end{aligned}
$$

where erf is the error function. We see that the error in having the strong pattern as a fixed point of the network is decreased in linear proportion to the degree of the strong pattern in the argument of error function. Moreover, the storage capacity or the load parameter $p / N \approx 0.138$ of the standard Hopfield network can now be increased by a factor of $d_{1}^{2}$, which will not change the argument of the error function, keeping the strong pattern still stable. In [33], it is in fact shown by considering the stochastic Hopfield network as the pseudo-temperature goes to zero that the storage capacity of the deterministic Hopfield network in the presence of a single pattern of degree $d_{1}$ is of the order of $0.138 d_{1}^{2}$. This square law property provides justification for using strong patterns to model attachment types and behavioural prototypes in psychology and psychotherapy.

In [32], [33], in order to use the Hopfield network as a model for attachment and behavioural prototypes, the creation of a strong pattern is identified as the formation of a prototype and the increase in the degree of the strong pattern as the strengthening of the prototype, which takes place as a result of repetition of training, corresponding to long term potentiation. For example consider the following experiment. We store a sad face multiple times in a network of size $N=50 \times 50$ and in addition store a large number $p$ of random images. This network is now meant to provide a simple model for a brain that is biased toward sadness. As the multiplicity of the storage of the sad face increases, the network would with higher probability retrieve the sad face when it is exposed to any random face. If the number of random stored patterns reaches or goes beyond $0.138 N=0.138 \times 2500 \approx 350$, then almost certainly the sad face is retrieved even if the network is exposed to one of the random stored patterns. Now consider the network when the sad face has been stored 30 times. The sad face will now remain retrievable if we store up to 30,000 random faces in the network. We now store a happy face in the network and gradually increase its multiplicity to go above 30. As soon as the multiplicity of the happy face exceeds that of the sad face, it would become more likely that the happy face is retrieved by any initial random pattern. Change of attachment or behavioural types has been modelled in this way in [32], [33]. It can also be used to model the process of psychotherapy in which a more positive cognitive and emotional prototype is gradually entrenched by repetition and will eventually outweigh an existing negative prototype.

In fact, a Hopfield network with strong patterns, representing the basic emotions and mentalisation, was used in [34], in combination with Levine's pathways for emotional-cognitive decision making and reinforcement learning to develop a quantitative model of cognitive behavioural therapy (CBT). The idea is that by reinforcement learning in the process of CBT, which rewards mentalisation, the brain becomes increasingly habituated by long term potentiation to make thoughtful rather than automatic and emotional decisions and this will allow the individual to reduce its negative affects.

In this paper we change our viewpoint and consider the degree of a pattern in the generalised Hebbian rule, Equation 1, as its strength which can now be a real number. The strength of a pattern can increase not just as a result of repetition of training but also as a result of any dopamine secretion associated with the pattern under consideration. This allows us to use the network to model any passionate pursuit or bond making as the creation of a strong pattern and retaining or strengthening the passionate pursuit or bond as increasing the strength of that pattern. In this way, we are able to develop a basic neural model for self-attachment therapy. We will however first describe the proposed self-attachment technique and its protocols. 


\section{SELF-ATTACHMENT TO TACKLE CHRONIC DEPRESSION AND ANXIETY}

We will briefly describe a proposed set of protocols for self-attachment to counter chronic depression and anxiety in adults. It is hypothesised that these protocols regulate and increase dopamine, serotonin, oxytocin and vasopressin and reduce cortisol levels in a natural way and create, by long term potentiation through repetition, new neural pathways that can reduce negative emotions and maximise positive affects, leading in particular to a more optimal development of the OFC, which as we have seen in Section II plays a crucial role in secure attachment.

Taking an attachment theory approach, our assumption is that the chronic symptoms of depression and anxiety are rooted in insecure attachment and grossly suboptimal and traumatic conditions in childhood such as prolonged child abuse, severe neglect or domestic violence. The notion of complex posttraumatic stress disorder or complex PTSD has been developed to explain the symptoms of individuals with such a prolonged period of childhood trauma or those who have suffered tortue and long periods in harsh conditions in prisons or concentration camps [35], [36].

Although existing psychotherapeutic techniques can help alleviate these symptoms to some extent, the sense of inner emptiness and helplessness deep rooted in these individuals usually persists. SSRI tablets in these individuals act by coreleasing serotonin and dopamine [37] which can help reduce the depression and anxiety but they can also produce sideeffects; invariably the symptoms can recur if medication is stopped [38].

As a self-therapy, the proposed secure self-attachment method aims to help the individual, henceforth called the volunteer, acquire the consistent and unconditional love and attention she or he had been deprived of in childhood.

The volunteer is imagined to consist of an inner child, representing the emotional self, rooted mostly in the right brain and the limbic system, which becomes dominant under stress, and an inner adult corresponding to the logical self, rooted mostly in the left brain and the prefrontal cortex, which is dominant in the absence of stress. We note that the notions of an inner child and an inner adult in modern psychotherapy originate from transactional analysis [39] and popular psychotherapy [40].

The proposed self-intervention consists of simulating the early right brain to right brain interactions between a securely attached infant and a good enough primary caregiver, by means of left brain to right brain interactions within the volunteer. Visual, verbal and tactile techniques are used to emulate the real infant-parent interaction as close as possible in the imagined two components of the self, represented by the inner child and the inner adult.

Self-attachment is related to a range of concepts and treatments among the existing psychotherapeutic methods, including transactional analysis [39], mentalisation [41], the capacity to understand the emotions and mental states of the other people as well as those of oneself, exposure as in behavioural therapy [42], compassionate focused psychotherapy [43], reparenting [3] and object-relation psychodynamic therapy in a wider sense of the term in which objects can be impersonal as well as personal [20]. Self attachment integrates these techniques into its main focus of intervention, which is the creation of an internal affectional bond to emulate what occurs naturally between an infant and a parent. Selfattachment also employs protocols such as singing, dancing and massage that are known to increase dopamine, serotonin, oxytocin and reduce cortisol levels [44], [45], [46], [47]. It can also be combined with any well-established therapeutic technique.

\section{A. Summary of Self Attachment Protocols}

There are four stages in self-attachment which are briefly described below:

(i) Introduction to secure self-attachment therapy. In the first stage, the volunteers study the scientific basis and the underlying hypothesis of the proposed self-attachment therapy, namely attachment theory and its neural correlates, fMRI studies on "falling in love" including in the case of abstract and imaginative objects as in prayer, plasticity and long term potentiation. This introduction is essential in order to provide in the volunteers the motivation and self-discipline required to practice the protocols which may sound unusual and may take a great deal of dedication before they can have an impact.

(ii) Connecting with the inner child. In this preparatory stage, the volunteers construct a basic psychological portrait of their inner child by looking at photos of their childhood and recalling their basic childhood environment such as the quality of relationships with parents and other care-givers. They also choose one happy or positive looking photo of their childhood that they have always favoured as well as a sad or gloomy looking photo that they have disliked or been less fond of. Then several exercises are practiced to "connect to the inner child" in various emotional states. These include trying to visualise the two chosen childhood photos with their eyes closed, to imagine with their eyes closed that the child that they were is present and is close to them and that they can touch and hold this child. The objective of the this stage is to conceptualise the "inner child" and feel compassionate toward it.

(iii) Falling in love with the inner child. In the third stage the volunteers create an imaginative but passionate affectional bond with the inner child which is subjectively experienced as "falling in love". It is hypothesised that based on "primary narcissism" in all human beings this step can be imaginatively taken. They subsequently adopt the inner child imaginatively in an emotional ceremony and vow to re-raise and reparent it. This task is facilitated by the volunteers choosing a favourite happy love song (e.g. Louis Armstrong's What a wonderful world) and singing it passionately, loudly and repeatedly while looking at the positive looking childhood photo. They imagine that by means of this singing they would rekindle the happy memories of their childhood and re-energise their inner child. It is hypothesised that the bond-making process with the inner child induces release of dopamine, serotonin and oxytocin, and reduces cortisol levels as in the happy interactions of an infantparent dyad [7]. By activating the reward system of the brain and reducing negative affects, hope and motivation are created in the volunteers to continue with the rest of the protocols in the anticipation of optimal emotional regulation which can overcome their feelings of inner emptiness and helplessness. 
(iv) Developmental training and re-parenting the inner child. The forth stage comprises the core of self-attachment practice in that it emulates the function of a securely attached parent in interacting with a child in order to carry out two tasks, as explained in Section II: minimise the negative emotions, referred to as the Sad-Child protocol, and maximise the positive affects, referred to as the Happy-Child protocol.

To process painful and traumatic past events, the volunteers with their eyes closed recall e.g. a traumatic scene in their childhood, remembering as much as possible the associated emotions such as terror, helplessness, humiliation and rage. Then, they imagine that their inner adult quickly approaches the child and embraces, cuddles, reassures and supports the child, thereby role playing good parents when they see their children in distress. Reassuring the inner child is carried out loudly and firmly while cuddling the inner child is simulated by giving oneself a head, face or neck massage. These exercise sessions, repeated over and over again, revisit neural circuits of past traumas. It is hypothesised that, by inducing dopamine, oxytocin and vasopressin secretion, they build new optimal neural circuits in relation to the old ones.

To regulate current negative emotions such as anger, rage, fear, anxiety or depression, in relation to every day life, the volunteers with closed eyes, imagine the unhappy childhood photo and project their negative emotions to this photo which represents the unhappy inner child. By projecting their negative emotions onto the inner child in this way, the volunteers make contact with their adult who based on their declared vow to look after the inner child now feel responsible to attend to the problems of the inner child, supporting the child and containing the child's negative emotions. While projecting their negative emotions to the unhappy photo and the inner child, the volunteers with eyes closed loudly reassure the inner child and, to simulate a cuddle for the inner child, give their own face a massage. Reassuring and cuddling continues until the volunteers contain the negative emotions and can switch from imagining the unhappy photo to focusing on the happy photo.

In order to maximise positive affect, the volunteers loudly and repeatedly recite with their whole body one or two examples of the love songs selected in the previous stage until the songs are learned by heart and reciting them many times a day becomes habituated. Thereby the bond with the inner child is retained and strengthened. Habituation to singing the selected love songs is so much enforced in this way that the volunteers develop a tendency to spontaneously and effortlessly engage in reciting these songs wholeheartedly and passionately. They gradually combine the singing with dancing with the imagined child including by looking into a mirror. They would eventually reach the position that they unintentionally recite these songs even when they are depressed or anxious, an activity which would alleviate their pain.

In the long term, volunteers learn to integrate the protocols of the forth stage into their every day life activities, such as by singing while walking or doing housework. In this way, the bond with the inner child is retained and thereby emotional regulation is sustained.

\section{B. Results of Case Studies}

The proposed Self attachment technique is in its infancy and there are only a few case studies and no study on a controlled trial. Beginning in 2010, a few individuals in the UK and US have practiced self attachment for various periods in the past four years. We have full data on two individuals with chronic depression and anxiety, who had previously tried several other techniques of therapy for long periods over several decades (including cognitive behavioural therapy, psychoanalytical therapy, yoga, mindfullness meditation and neurofeedback) but still suffered from their long term symptoms. After a few weeks of practicing self attachment they reported a transformative change, relief from inner emptiness and helplessness and for the first time they reported the experience of inner joy, and a sense of agency and mastery over their lives. Under high levels of stress, they assert, they still slide back temporarily to their disorders but with much reduced pain and duration. Since these painful periods become shorter and fewer in between, they have say that their lives have now changed for good.

Since April 2014, a number of professional psychotherapists have tried the self-attachment protocols on their clients. While the method was not offered by these therapists to all their clients and some clients chose not to undertake it, all those clients who actually tried the protocols reported significant improvement in a few weeks. However, all these studies have been uncontrolled and cannot be the basis of any objective evaluation. We intend to carry out controlled studies in the near future.

\section{A Basic NeUral Model of Self-Attachment}

We start by reviewing several neural models of psychotherapy in the literature. In [48], a neural model for personality has been proposed based on Cloningers character cube [49], which, according to the authors, deals with individuals who are already functioning quite well but seek to improve their effectiveness. Similarly, the work in [50] uses Cloningers character cube to develop a neural model for how human beings suppress or enhance certain types of behaviour. Galatzer-Levy provides in [51], an expository account of how non-linear dynamical systems and attractors can be used qualitatively to model the psychoanalytical process; it also contains other related references in this subject. behavior. In [52], a Hopfield network with two weakly connected layers has been used to model the concept of working through in psychoanalysis.

In [53], a neural model of the internal bond making in stage (iii) of the self-attachment protocols has been presented. In this section, we use the notion of strong patterns presented in Section V and the work in [34] developed for modelling mentalisation based psychotherapy to construct a model of stage (iv) protocols for Happy-Child and Sad-Child corresponding to interactions with the happy child and the sad child in self attachment.

The framework in [34] is based on Levine's pathways for emotional-cognitive decision making [54], which contains two networks: An energy based competitive needs network that is inspired by Maslow's hierarchy of needs and includes physiological as well as higher needs and a network of decision rules with four connected areas: the amygdala, the OFC, ACC and 
DLPFC, which account for various decision rules on specific tasks. These four regions comprise a three-layer network, in which the vigilance threshold of the individual determines the status of activation of each layer. The state of the needs network influences the vigilance threshold represented by ACC so that the winning needs become dominant to implement the corresponding decision rules.

The framework in [34] employs a form of reinforcement learning, called Q-learning, for rewarding mentalisation in a competitive Hopfield network of needs that in particular consists of the two categories of (a) need for cognitive closure, which includes the six basic emotions, and (b) need for cognition, which contains mentalisation. Strong patterns with different degrees each represent the six basic emotions and mentalisation in the needs network which also contains a large number of random patterns (we used 500 in our experiment). The six basic emotions and the mentalisation pattern are represented by generic smiley faces. Three identical Restricted Boltzmann Machines with 17 hidden units are pre-trained to recognise the six emotions and the mentalisation pattern and are used to model the amygdala, the OFC and the DLPFC. We refer the reader to [34] for the details.

We adopt this framework here for self-attachment by extending the category of need for cognition with two additional strong patterns namely the protocols for Happy-Child and SadChild. When the brain is exposed to a random stimulus one of the strong patterns, most probably the one with highest degree, is recalled in the Hopfield network. If the mentalising pattern, the Happy-Child or Sad-Child is recalled, then the Hopfield network will send a low level vigilance threshold to the error detector (ACC), and the DLPFC-OFC loop is chosen to generate complex decision rules, which includes practicing the protocols for Happy-Child or Sad-Child. As the secondary sensory device, the RBM in the DLPFC receives a mentalising signal from the Hopfield network, and categorises it into a 17-unit vector. In addition, the RBM in the OFC, the primary sensory device, accounts for categorising the input pattern into another 17-unit vector. If the Hamming distance of these two generated vectors is greater than the vigilance threshold, then we say that a mismatch occurs and the network generates a deliberative rule. Otherwise, a heuristic decision is made. However, because of the low vigilance threshold, the DLPFCOFC loop would most likely not make heuristic decisions in this case.

If, on the other hand, the recalled pattern is one of the six emotion patterns, the Hopfield network will send a high-level vigilance threshold to ACC, and the OFC-amygdala loop is chosen for making decisions. Similar to the loop above, as the secondary sensory device, the RBM representing the OFC categorises the emotion pattern from the Hopfield network, and amygdala categorises the input pattern. The Hamming distance between the two 17 unit output vectors is compared to the high-level vigilance threshold. A heuristic decision is generated if the Hamming distance (i.e, the number of components that are different in the two vectors) is lower than the vigilance threshold; otherwise, a deliberative decision is made. Furthermore, the control from the OFC to amygdala influences the output of the RBM in amygdala. A well-trained OFC can adjust the amygdala output to some value close to the output of the OFC.
The Q-state of the Hopfield network is given by the ordinal representation, i.e., the relative weight, of the degrees of the 9 strong patterns present. Corresponding to the nine strong patterns, there are nine related actions such as be-happy, beangry or do-mentalising, do-Happy-Child and each pair $(s, a)$ of a Q-state $s$ and an action $a$ has a real number $Q(s, a)$ as its Q-value. The Q-value for each state-action pair is updated in the course of iteration of the network according to the Qlearning rule based on the reward for choosing the action in that state:

$$
Q(s, a) \leftarrow Q(s, a)+\lambda\left(R_{a}\left(s, s^{\prime}\right)+\gamma\right)
$$

where $R_{a}\left(s, s^{\prime}\right)$ is the reward when in state $s$ action $a$ is taken to reach state $s^{\prime}$, the parameter $\lambda$ is the learning rate, and $\gamma=\delta \max _{a^{\prime}} Q\left(s^{\prime}, a\right)-Q(s, a)$, with $\delta$ as the discount factor. We have made $R_{a}\left(s, s^{\prime}\right)$ only dependent on the action $a$, and have set $\lambda=1$ and $\delta=0.2$ in our model.

In our implementation, in addition, we have restricted the basic emotions whose degrees are updated in the network to Angry, Happy and Sad so that we have a total of six strong pattens, which include Mentalising, Happy-Child and SadChild. The following values have been taken as rewards for the set $A$ of actions:
Angry: 0
Happy: 0.3
Sad: 0
Mentalising: 0.4

\section{Happy-Child: $1 \quad$ Sad-Child: 0.6}

Since the practice of self attachment protocols Happy-Child and Sad-Child induce, according to our hypothesis, dopamine, serotonin or oxytocin, which result in hope, positive affect or calm, the rewards for these two protocols are set higher than mentalising.

The Q-value of a state-action pair determines the probability of choosing the given action in the given state in the pair according to the Boltzmann rule:

$$
P(a \mid s)=\frac{k^{Q(s, a)}}{\sum_{a \in A} k^{Q(s, a)}}
$$

where $k>1$ is the exploration factor, which we have set to $k=2$.

In a course of therapy, which starts by a dominant strong pattern for anger, every time a rewarding action is selected in a Q-state, the state-action pair is rewarded and is therefore more likely that the given rewarding action is selected in future in that given state. Moreover, every time an action is selected the degree of the strong pattern for that action is incremented by one unit. In a successful course of therapy, the Happy-Child will eventually become the more dominant pattern outperforming the angry pattern that had been initially dominant. The brain network makes decisions that are more deliberate and thoughtful than heuristic and automatic, and decisions that result in more positive affect. The network as a whole is depicted in Figure 1.

In a single experiment, the patient chooses an action subject to the reinforcement rules, and then the Hopfield network 

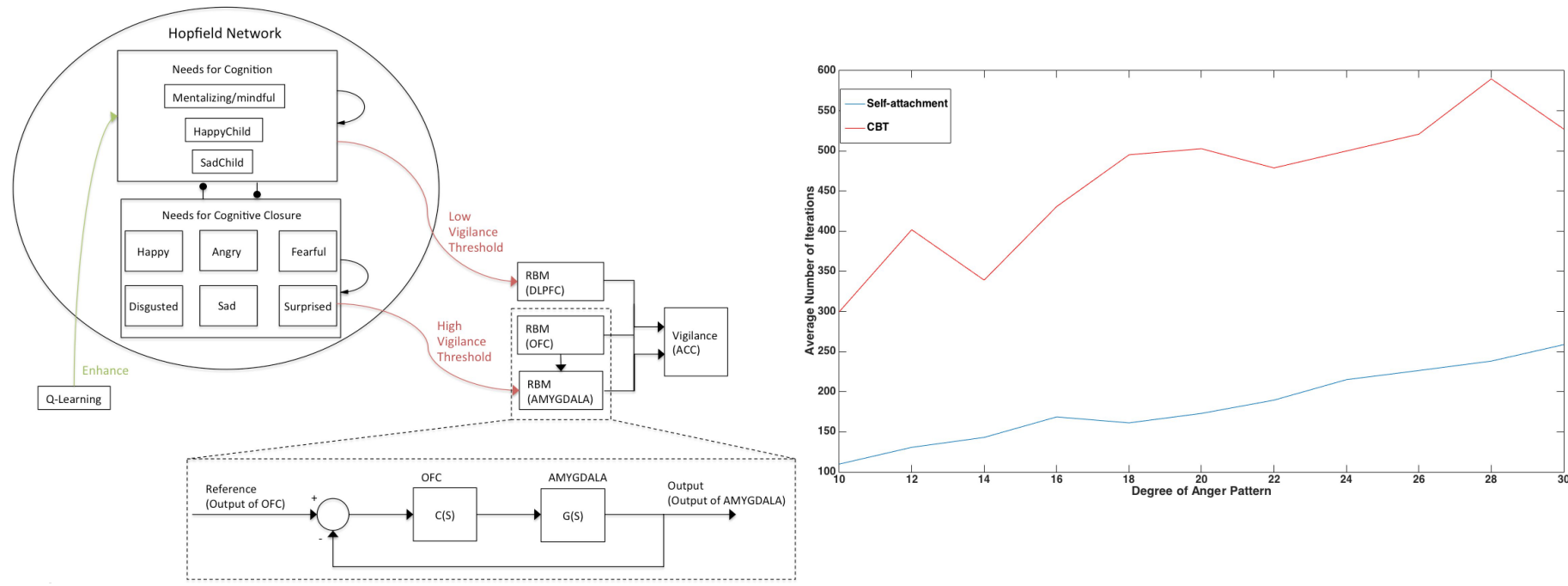

Fig. 1. A neural model for self attachment combining a Hopfield network as a needs network, and RBMs as a decision network. Q-learning influences the attractors in the Hopfield network. Needs for cognitive closure, represented by the six basic emotions, output high-level vigilance threshold signals to ACC. Needs for cognition, including mentalisation and two protocols of self attachment, Happy-Child and Sad-Child output low-level vigilance threshold signals to ACC. The control from OFC to amygdala is designed as a feedback control loop.

is updated according to the chosen action. Subsequently, a random pattern is used to stimulate the Hopfield network and the output is recorded. This is considered as one iteration. Such iteration will be repeated 200 times in the single experiment. This experiment is run 1000 times and each time we have recorded the decisions that are made during the Q-learning procedure (200 iterations).

Figure 2 shows the average number of iterations required (blue) so that the Happy-Child becomes the dominant pattern in the self attachment network when the initial state has different degrees for the dominant Angry strong pattern. This is compared to the performance of a therapeutic model that only contains mentalisation, e.g. cognitive behavioural therapy (CBT), i.e., the average number of iterations required (red) so that the Mentalisation pattern becomes dominant. The figure shows that on average the self-attachment protocols achieve their goal 2 to 4 times faster than mentalisation alone.

In both experiments, we have incremented the degree of a strong pattern by one unit if the action corresponding to that pattern is selected. Since, however, we have hypothesised that the self-attachment protocols increase the dopamine level in the individual, it is reasonable to assume that learning is faster and more effective when the self-attachment protocols in particular the Happy-Child is selected. Therefore its degree or strength should be increased by more than one unit, which means that the convergence to the state in which the HappyChild dominates the Hopfield network would be much faster. We will present our simulation results with this choice in the full version of the paper.

Fig. 2. Blue curve: The average number of iterations required so that, starting with the angry dominant pattern with different degrees, the Happy-Child strong pattern becomes dominant. Red curve: The average number required when only mentalisation is in the need for cognition, i.e., without the self attachment protocols.

\section{CONCLUSION}

We have introduced the inter-disciplinary concept of selfattachment based on the common observed pattern in the fMRI studies of "falling in love" in three different contexts, on evidence from developmental psychology and psychology of religion, and on the behaviour of strong patterns in energy based neural networks. Self attachment can be viewed as an overarching notion that integrates similar experiences from different areas. Motivated by these similarities, we have introduced a proposed psychotherapeutic method, namely selfattachment, whose focus of intervention is based on creating an internal affectional bond within the individual. The individual then practices various protocols which by long term potentiation construct new neural circuits that regulate emotions and arousal level, reducing negative emotions and increasing positive affect. While initial case studies by volunteers and independent professional psychotherapists have been promising, organising controlled trials of the technique is now a high priority. Based on these trials we need to evaluate the scope and efficacy of the technique and determine what type of disorders in what type of individuals would respond positively to it. A more refined neural model of self attachment that can integrate the work in [53] on bond making and the ideas presented here as a neural model for the practice of the protocols will be required to be developed.

\section{ACKNOWLEDGEMENT}

I would like to thank Homa Noshirvani, Kamran Saedi, Peter Fonagy, Hosein Djalilian, Felicity de Zulueta, Shiva Dolatabadi, Hassan Ashayeri, Maryam Rasoulian and Anna Witham for their comments and support for this research. Thanks also to Zheng Lin who ran the simulations and David Cittern who read the first draft of the paper.

\section{REFERENCES}

[1] P. Fonagy, Attachment Theory and Psychoanalysis. Other Press, 2001. 
[2] A. Schore, The Science of the Art of Psychotherapy. W. W. Norton and Co., 2012.

[3] J. E. Young, J. S. Klosko, and M. E. Weishaar, Schema therapy: A practitioner's guide. Guilford Press, 2003.

[4] A. Edalat, "Self-attachment: A new and integrative psychotherapy," 2013, Talk at the Institute of Psychiatry, London, 02-05-2013.

[5] M. H. Van Ijzendoorn and P. M. Kroonenberg, "Cross-cultural patterns of attachment: A meta-analysis of the strange situation," Child Development, pp. 147-156, 1988.

[6] A. N. Schore, Affect Dysregulation and Disorders of the Self. W. W. Norton, 2003.

[7] L. Cozolino, The Neuroscience of Human Relationships. W. W. Norton, 2006.

[8] R. N. Emde, "Development terminable and interminable: Ii. recent psychoanalytic theory and therapeutic considerations." The International Journal of Psychoanalysis, 1988.

[9] J. D. Ford, "Neurobiological and developmental research," Treating complex traumatic stress disorders: An evidence-based guide, pp. 3158, 2009.

[10] L. I. Perlovsky, Neural dynamic logic of consciousness: the knowledge instinct. Springer, 2007.

[11] M. M. Riem, M. J. Bakermans-Kranenburg, M. H. van IJzendoorn, D. Out, and S. A. Rombouts, "Attachment in the brain: adult attachment representations predict amygdala and behavioral responses to infant crying," Attachment \& human development, vol. 14, no. 6, pp. 533551, 2012.

[12] A. Bartels and S. Zeki, "The neural basis of romantic love," Neuroreport, vol. 11, no. 17, pp. 3829-3834, 2000.

[13] A. Aron, H. Fisher, D. J. Mashek, G. Strong, H. Li, and L. L. Brown, "Reward, motivation, and emotion systems associated with early-stage intense romantic love," Journal of neurophysiology, vol. 94, no. 1, pp. 327-337, 2005.

[14] A. Bartels and S. Zeki, "The neural correlates of maternal and romantic love," Neurolmage, vol. 21, pp. 1155-1166, 2004.

[15] S. U. and Stdkilde-Jrgensen H. and Geertz A. W. and Roepstorff A., "Rewarding prayers," Neurosci Lett., vol. 443, no. 3, pp. 165-8, October 2008.

[16] U. Schjoedt, "It's a brain puzzle," 2012, http://www.theeuropeanmagazine.com/649-schj-dt-uffe/650-the-neuroscience-of-prayer.

[17] D. Winnicott, “(1953). international journal of psycho-analysis, 34: 8997 transitional objects and transitional phenomenaa study of the first not-me possession1," International Journal of Psycho-Analysis, vol. 34, pp. 89-97, 1953 .

[18] C. J. Litt, "Theories of transitional object attachment: An overview," International Journal of Behavioral Development, vol. 9, no. 3, pp. 383-399, 1986.

[19] D. W. Winnicott and M. M. R. Khan, The maturational processes and the facilitating environment: Studies in the theory of emotional development. Hogarth Press London, 1965.

[20] A. Storr, Solitude. Flamingo, 1988.

[21] W. W. Meissner, Psychoanalysis and religious experience. Yale University Press, 1986.

[22] K. Armstrong, A History Of God. Vintage, 1990.

[23] W. James, The varieties of religious experience. Harvard University Press, 1985, vol. 15.

[24] F. C. Van der Horst, H. A. LeRoy, and R. Van der Veer, "when strangers meet: John bowlby and harry harlow on attachment behavior," Integrative Psychological and Behavioral Science, vol. 42, no. 4, pp. 370-388, 2008

[25] J. P. Capitanio, "Behavioral pathology," in Comparative Primate Biology, G. Mitchell and J. Erwin, Eds. Alan R. L iss, 1986, vol. Volume 2A: Behavior, Conservation, and Ecology, pp. 411-454.

[26] D. Petters, "Building agents to understand infant attachment behaviour," International Joint Conference on Artificial Intelligence, vol. , no. , pp. $158-165,2005$,

[27] A. Hiolle, M. Lewis, and L. Cañamero, "Arousal regulation and affective adaptation to human responsiveness by a robot that explores and learns a novel environment," Frontiers in neurorobotics, vol. 8, 2014.
[28] M. A. Cohen and S. Grossberg, "Absolute stability of global pattern formation and parallel memory storage by competitive neural networks," Systems, Man and Cybernetics, IEEE Transactions on, vol. SMC-13, no. 5, pp. 815-826, 1983.

[29] D. Cittern and A. Edalat, "An arousal-based neural model of infant attachment," in Proceedings of IEEE Symposium Series on Computational Intelligence, 2014, iEEE SSCI 2014.

[30] T. Lewis, F. Amini, and R. Richard, A General Theory of Love. Vintage, 2000.

[31] S. C. Thomas S. Smith, Gregory T. Stevens, "The familiar and the strange: Hopfield network models for prototype-entrained attachmentmediated neurophysiology," in Mind, brain, and society: Toward a neurosociology of emotion, T. S. E. Franks, David D. (Ed); Smith, Ed. Elsevier Science/JAI Press, 1999, pp. 213-245.

[32] A. Edalat and F. Mancinelli, "Strong attractors of Hopfield neural networks to model attachment types and behavioural patterns," in IJCNN 2013 Conference Proceedings. IEEE, August 2013.

[33] A. Edalat, "Capacity of strong attractor patterns to model behavioural and cognitive prototypes," in Advances in Neural Information Processing Systems 26, C. Burges, L. Bottou, M. Welling, Z. Ghahramani, and K. Weinberger, Eds. ACM, 2013.

[34] A. Edalat and Z. Lin, "A neural model of mentalization/mindfulness based psychotherapy," in Neural Networks (IJCNN), 2014 International Joint Conference on. IEEE, 2014, pp. 2743-2751.

[35] J. L. Herman, "Complex ptsd: A syndrome in survivors of prolonged and repeated trauma," Journal of traumatic stress, vol. 5, no. 3, pp. 377-391, 1992.

[36] B. A. Van der Kolk, The body keeps the score. Viking, 2014.

[37] F.-M. Zhou, Y. Liang, R. Salas, L. Zhang, M. De Biasi, and J. A. Dani, "Corelease of dopamine and serotonin from striatal dopamine terminals," Neuron, vol. 46, no. 1, pp. 65-74, 2005.

[38] N. C. C. for Mental Health (UK et al., Depression: the treatment and management of depression in adults (updated edition). British Psychological Society, 2010.

[39] I. Stewart and V. Joines, TA today: A new introduction to transactional analysis. Lifespace Pub., 1987.

[40] C. Whitfield, Healing the Child Within. Health Communications, 1991.

[41] J. Allen, P. Fonagy, and A. Bateman, Mentalizing in Clinical Practice. American psychiatric Publishing, 2008.

[42] J. S. Abramowitz, B. J. Deacon, and S. P. Whiteside, Exposure therapy for anxiety: Principles and practice. Guilford Press, 2012.

[43] P. Gilbert, Compassion: Conceptualisations, research and use in psychotherapy. Routledge, 2004.

[44] B. Kleber, N. Birbaumer, R. Veit, T. Trevorrow, and M. Lotze, "Overt and imagined singing of an italian aria," Neuroimage, vol. 36, no. 3, pp. 889-900, 2007.

[45] K. Jeffries, J. B. Fritz, and A. R. Braun, "Words in melody: an h215o pet study of brain activation during singing and speaking," Neuroreport, vol. 14, no. 5, pp. 749-754, 2003.

[46] C. Q. Murcia, S. Bongard, and G. Kreutz, "Emotional and neurohumoral responses to dancing tango argentino the effects of music and partner," Music and Medicine, vol. 1, no. 1, pp. 14-21, 2009.

[47] T. Field, M. Hernandez-Reif, M. Diego, S. Schanberg, and C. Kuhn, "Cortisol decreases and serotonin and dopamine increase following massage therapy," International Journal of Neuroscience, vol. 115, no. 10, pp. 1397-1413, 2005.

[48] A. M. C. Aleksandrowicz and D. S. Levine, "Neural dynamics of psychotherapy: what modeling might tell us about us," Neural networks, vol. 18 , no. 5, pp. 639-645, 2005.

[49] C. R. Cloninger, "A new conceptual paradigm from genetics and psychobiology for the science of mental health," Australian and New Zealand Journal of Psychiatry, vol. 33, no. 2, pp. 174-186, 1999.

[50] D. S. Levine, "Angels, devils, and censors in the brain," ComPlexus, vol. 2, no. 1, p. 35, 2005.

[51] R. M. Galatzer-Levy, "Good vibrations: Analytic process as coupled oscillations," The International Journal of Psychoanalysis, vol. 90, no. 5, pp. 983-1007, 2009. 
[52] R. S. Wedemann, R. Donangelo, and L. A. de Carvalho, "Generalized memory associativity in a network model for the neuroses," Chaos: An Interdisciplinary Journal of Nonlinear Science, vol. 19, no. 1, p. 015116, 2009.

[53] D. Cittern and A. Edalat, "Towards a neural model of bonding in selfattachment," 2015, submitted to IJCNN 2015.

[54] D. S. Levine, "Brain pathways for cognitive-emotional decision making in the human animal," Neural Networks, vol. 22, pp. 286-293, 2009. 